\title{
EUS-guided gallbladder drainage using a lumen-apposing self-expandable metal stent in patients with coagulopathy or anticoagulation therapy: a case series $\square$
}

\section{(c) $(>)$}

\author{
Authors \\ Alberto Larghi \\ Institutions \\ 1 Department of Gastroenterology, Digestive Endoscopy \\ Unit, Humanitas Research Hospital, Rozzano, Italy \\ 2 Digestive Endoscopy Unit, Catholic University, Rome, \\ Italy \\ 3 Gastroenterology Department, Colentina Clinical \\ Hospital, Carol Davila University of Medicine, Bucharest, \\ Romania \\ 4 IHU-USIAS, University of Strasbourg, France
}

Andrea Anderloni ${ }^{1}$, Fabia Attili ${ }^{2}$, Alessandro Sferrazza ${ }^{1}$, Mihai Rimbaș ${ }^{2,3}$, Guido Costamagna ${ }^{2,4}$, Alessandro Repici ${ }^{1}$,

submitted 11.4.2017

accepted after revision 19.6.2017

Bibliography

DOI https://doi.org/10.1055/s-0043-118828 |

Endoscopy International Open 2017; 05: E1100-E1103

(c) Georg Thieme Verlag KG Stuttgart · New York

ISSN 2364-3722
Corresponding author

Andrea Anderloni MD, PhD, Digestive Endoscopy Unit, Division of Gastroenterology, Humanitas Research Hospital, Via Manzoni 56, 20089 Rozzano (Milano), Italy

Fax: +39-02-82247308

andrea.anderloni@humanitas.it

\section{ABSTRACT}

Background and study aims We report our experience in endoscopic ultrasound-guided gallbladder drainage performed emergently in 4 prohibitive surgical risk patients with concurrent coagulopathy or on anticoagulant therapy using an electrocautery enhanced lumen apposing self expanding metal stent (LA-SEMS).

\section{Introduction}

Endoscopic ultrasound-guided gallbladder drainage (EUS-GBD) is becoming a valid alternative to percutaneous transhepatic gallbladder drainage (PTGBD) in high-risk surgical candidates. A randomized controlled trial reported that EUS-GBD using a naso-biliary catheter is comparable to PTGBD in terms of technical feasibility, clinical efficacy, and adverse event $(A E)$ rates [1]. In addition, 2 systematic reviews of all EUS-GBD cases have shown technical and clinical success rates greater than $95.8 \%$ and $93.4 \%$, respectively $[2,3]$.

Recently, a lumen-apposing fully-covered self-expanding metal stent (LA-FCSEMS) with bilateral flanges specifically designed to create an anastomosis, the AXIOS ${ }^{\mathrm{TM}}$ stent (Boston Scientific Corp., Marlborough, MA, USA), has become available. More recently, the stent has been incorporated in an electrocautery- enhanced delivery system, the AXIOS-EC'M (Boston Scientific Corp.) [4], which could render the procedure faster and safer by decreasing the number of accessory exchange needed to accomplish the drainage. Reports of use of the stent with and without the electrocautery-enhanced delivery system have been reported for drainage of pancreatic fluid collections [5], common bile duct [6] and gallbladder [7].

No data on possible use of and AEs with the device in patients on anticoagulants or with coagulopathy who undergo drainage have been published up to now. We report our experience with endoscopic treatment of 4 prohibitive surgical risk patients with acute cholecystitis and concurrent coagulopathy or on anticoagulant therapy.

A therapeutic linear echoendoscope (GF-UC180T, Olympus Medical System Europe, Hamburg, Germany) was used in all cases. The drainage site was chosen based on where the gallbladder was best visualized from the stomach or the duodenum. The AXIOS-ECTM device was advanced through the working channel of the echoendoscope and pushed outside the tip of the scope until a contact with the wall of the gastrointestinal (GI) tract was achieved. In all 4 patients described, the drainage was performed using the AXIOS-EC ${ }^{\mathrm{TM}}$ (Boston Scientific Corp.) containing a $10-\mathrm{mm}$ wide, 10-mm long LA-SEMS (> Fig.1). Penetration of the electrocautery-enhanced device into the gallbladder was achieved by appling pure cut current, with the setting adjusted to 100 watts, without coagulation (ICC 200, 


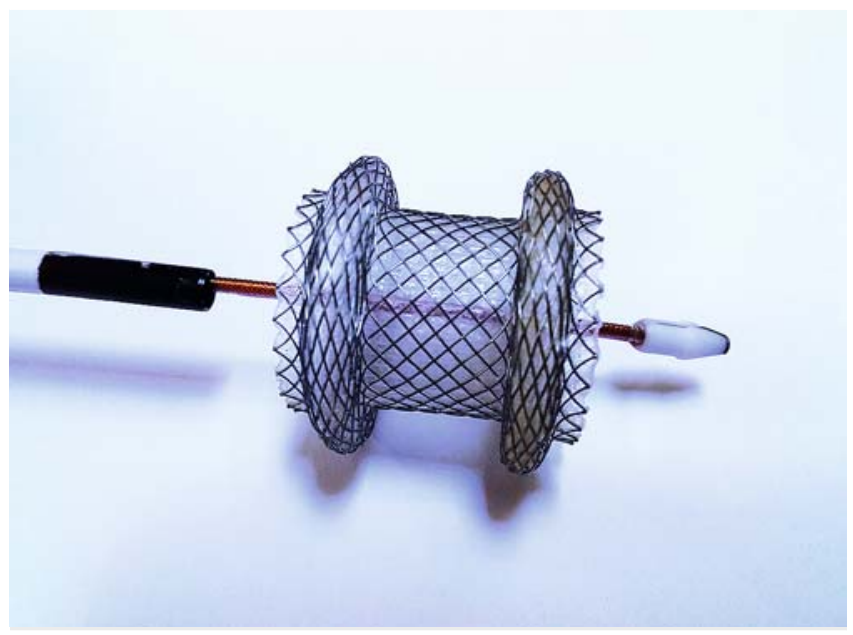

- Fig. 1 The novel electrocautery-enhanced delivery system with the lumen-apposing self expanding metal stent fully opened (AXIOS-ECTM, Boston Scientific Corporation).

ERBE Elektromedizin GmbH, Tübingen, Germany). Once the delivery catheter was inside the gallbladder, the stent was deployed completely under EUS guidance as previously described $(\triangleright$ Video 1 and $\triangleright$ Fig. 2) [8].

\section{Case Reports}

\section{Patient 1}

A 55-year-old male developed acute cholecystitis after cardiac surgery. After 3 days of broad-spectrum antibiotics, his clinical condition worsened and the patient became septic with hypotension. EUS-GBD was performed despite the international normalized ratio (INR) value of 1.67 due to multisystem organ failure secondary to sepsis. The patient was also on active anticoagulation receiving enoxaparin sodium $6,000 / \mathrm{Ul}$ twice a day,

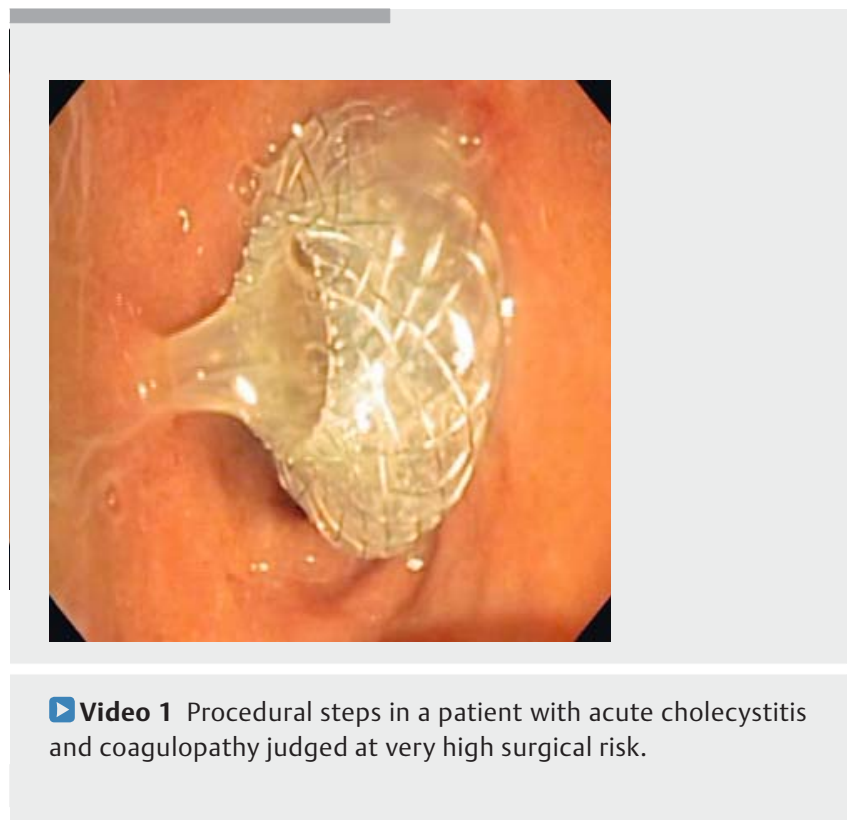

which could not be withheld. Gallbladder drainage was done transgastrically, without any immediate or late procedurerelated AEs. However, the patient died three days after the procedure due to sepsis and infectious endocarditis.

\section{Patient 2}

A 90-year-old female was referred for jaundice (serum bilirubin $4.9 \mathrm{mg} / \mathrm{dL}$ ), increased white blood cell (WBC) count $\left(13,200 / \mathrm{mm}^{3}\right)$ and suspicion of acute cholecystitis (right upper quadrant pain for more than 24 hours and fever $>38^{\circ} \mathrm{C}$ ). Transabdominal ultrasound showed hydrops of the gallbladder with multiple stones. The day after she was not responding to the antibiotics, thus an emergent EUS-GBD was done. At the time of the procedure the INR was 1.53 and she was receiving $2.5 \mathrm{mg}$ of fondaparinux sodium injection twice a day with the last dose of anticoagulant administered 6 hours before the endoscopic procedure. EUS-GBD was performed from the stomach. No procedure-related AEs were encountered. The patient recovered completely and was discharged 10 days after the procedure.

\section{Patient 3}

A 63-year-old female with metastatic breast cancer was referred for jaundice (serum bilirubin $28.3 \mathrm{mg} / \mathrm{dL}$ ), increased WBC count $\left(31,800 / \mathrm{mm}^{3}\right)$ and suspicion of acute cholecystitis. An abdominal computed tomography scan revealed a distended gallbladder and liver metastases causing compression of the cystic duct. Endoscopic retrograde cholangiopancreatography failed because of difficulty in reaching the papilla. The patient was then treated during the same endoscopic session with transgastric gallbladder drainage using the Axios-EC'M device. The day of the procedure the INR value was 2.41 presumably due to her liver failure and she was receiving enoxaparin sodium 6,000/UI twice a day, with the last dose of anticoagulant administered 2 hours before the procedure. No immediate procedure-related AEs were observed. The patient died two days after the procedure due to multisystem organ failure.

\section{Patient 4}

A 90-year-old male was admitted to the medical intensive care unit with fever, jaundice and abdominal pain. Blood testing revealed increased WBC count $\left(17,000 / \mathrm{mm}^{3}\right)$ and serum bilirubin level $(13.9 \mathrm{mg} / \mathrm{dl})$. Transabdominal ultrasound revealed a distended gallbladder, with wall thickening, microlithiasis, and peri-cholecystic fluid. The patient was started on antibiotics, but became septic with hypotension and disseminated intravascular coagulation (DIC) syndrome, which required emergent endoscopic transgastric gallbladder drainage to be performed. The day of the procedure the INR value was 3.5, presumably due to DIC. No immediate or late adverse procedure-related events were observed. The patient recovered completely and was discharged 7 days after the procedure. 

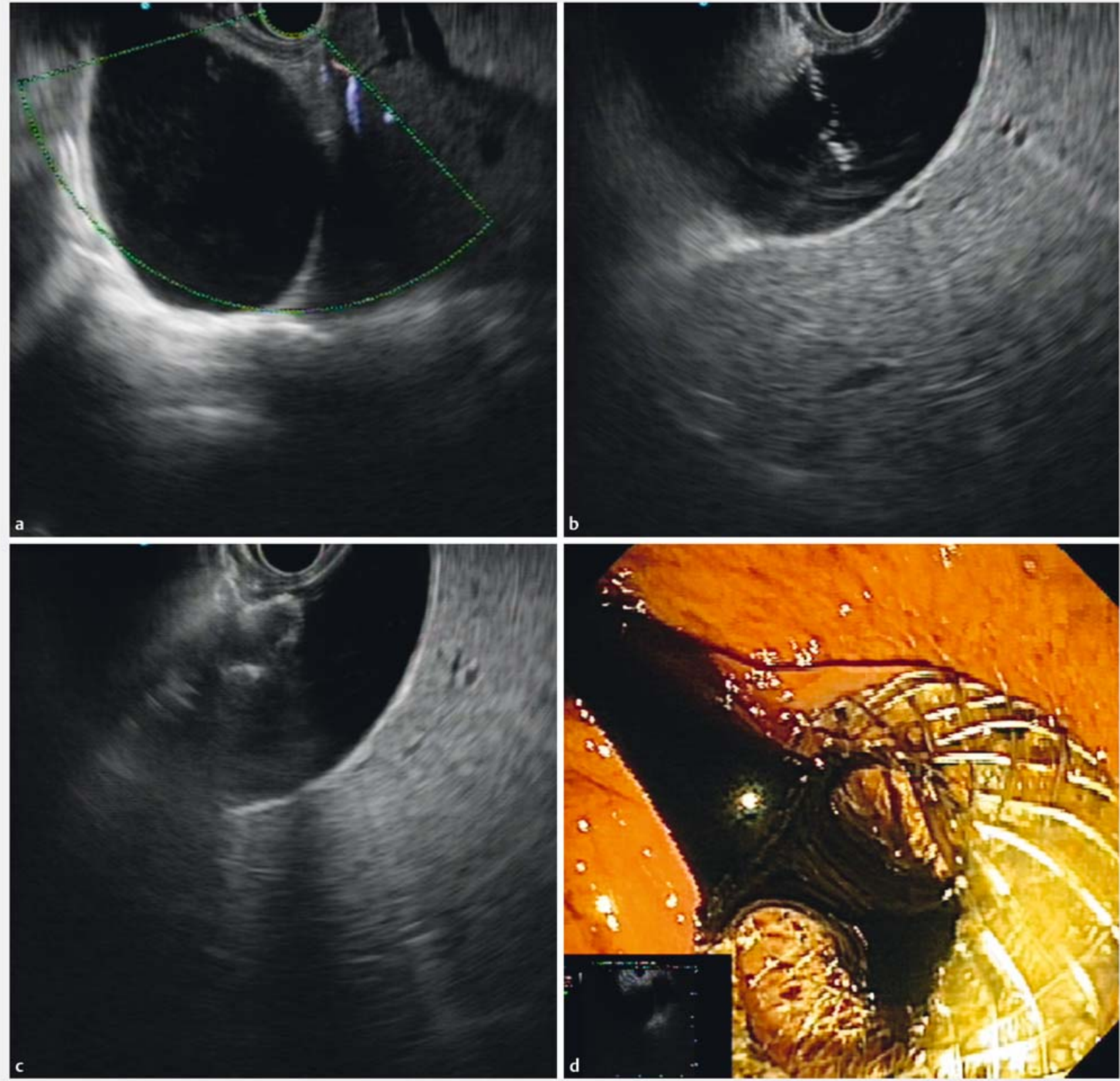

- Fig. 2 EUS-guided gallbladder drainage using a LA-SEMS in a patient with acute cholecystitis, high surgical risk, and INR of 2.41. a EUS image with the use of Doppler to select a target area with no visible vessels. $\mathbf{b}$ puncture with the electrocautery device performed under realtime EUS guidance. $\mathbf{c}$ EUS-guided deployment of the stent; $\mathbf{d}$ Endoscopic view of the stent deployed in the duodenum with dark bile flowing out of its proximal flange, with no bleeding.

\section{Discussion}

We herein report for the first time utilization of a recently developed lumen-apposing FCSEMS mounted on an electrocautery-enhanced delivery system to perform emergent EUS-GBD in 4 prohibitive surgical risk patients with acute cholecystitis with prolonged INR and/or who were taking anticoagulants. All stent placements were successful without any procedural or post-procedural bleeding. Unfortunately, 2 of the 4 treated pa- tients died 2 and 3 days after the procedure due to multiorgan failure, without any sign of bleeding.

Currently, there are no reccomandations regarding bleeding risk withr the drainage procedure accomplished with the newly developed LA-FCSEMS in patients with acute cholecystitis and high surgical risk with prolonged INR. According to current ESGE guidelines, a simple EUS with FNA in a patient with increased INR is considered a high-risk procedure. The guidelines recommend careful checking of the INR value prior to the procedure to ensure that it is less than 1.5 [9]. Moreover, if EUS- 
guided sampling has to be performed in a patient on low-molecular-weight heparin (LMWH), the procedure should be performed at least 8 hours after the last dose of the drug has been administered $[9,10]$, and it should be restarted only on the day after the procedure [9].

In our experience 3 of the 4 patients were actively receiving anticoagulants, which in one of them was continued also after the procedure, with no occurrence of bleeding. The INR value was slightly prolonged in 2 patients, and markedly prolonged in the other 2, precluding performance of an endoscopic interventional procedure as recommended by the ESGE guideline [9]. However, the alternative was percutaneous gallbladder drainage, which was judged to be even riskier than the endoscopic one.

The lack of procedural and post-procedural bleeding might be explained by 1 aspect of our process and 2 characteristics of the stent: (1) lack of prior use of an FNA needle, which is not used when performing the procedure with the one-step AxiosECTM technique; (2) the very small surface area of the electrode ( $3 \%$ of a $10 \mathrm{~F}$ cystotome), which makes the current density quite high and focuses the energy very precisely, thus causing very little thermal trauma; and (3) the radial expansion force of the fully covered stent, which is able to tamponade any bleeding vessel within the stent flanges [11]. Similarly, cases of postsphincterotomy, balloon sphincteroplasty and post-transplant biliary anastomotic stricture dilatation bleeding successfully treated using the expansion forces of a FC-SEMSs have been reported [12,13]. Importantly, if bleeding occurs after the procedure, transcatheter arterial embolization will be facilitated by presence of the metalic stent, which can act as a guide for the angiographer in identifiying the bleeding vessel [14].

\section{Conclusion}

In conclusion, our case series suggests that EUS-guided gallbladder drainage in patients with prohibitive surgical risk with concomitant prolonged INR and/or on anticoagulation is feasible without apparent increased bleeding risk. Importantly, that should be done in tertiary care centers with multidisciplinary teams available, in case of the occurrence of AEs, and the coagulopathy should be corrected or reversed, whenever possible.

\section{Competing interests}

Alberto Larghi, Alessandro Repici, and Guido Costamagna are consultants for Boston Scientific Corp.

\section{References}

[1] Jang JW, Lee SS, Song TJ et al. Endoscopic ultrasound-guided transmural and percutaneous transhepatic gallbladder drainage are comparable for acute cholecystitis. Gastroenterology 2012; 142: 805 811

[2] Peñas-Herrero I, de la Serna-Higuera C, Perez-Miranda M. Endoscopic ultrasound-guided gallbladder drainage for the management of acute cholecystitis (with video). J Hepatobiliary Pancreat Sci 2015; 22: $35-43$

[3] Anderloni A, Buda A, Vieceli F et al. Endoscopic ultrasound-guided transmural stenting for gallbladder drainage in high-risk patients with acute cholecystitis: a systematic review and pooled analysis. Surg Endosc 2016; 30: 5200 - 5208

[4] Teoh AY, Binmoeller KF, Lau JY. Single-step EUS-guided puncture and delivery of a lumen-apposing stent for gallbladder drainage using a novel cautery-tipped stent delivery system. Gastointest Endosc 2014; 80: 1171

[5] Rinninella E, Kunda R, Dollhopf M et al. EUS-guided drainage of pancreatic fluid collections using a novel lumen-apposing metal stent on an electrocautery-enhanced delivery system: a large retrospective study (with video). Gastrointest Endosc 2015; 82: 1039-1046

[6] Attili F, Rimbaş M, Galasso D et al. Fluoroless endoscopic ultrasoundguided biliary drainage after failed ERCP with a novel lumen-apposing metal stent mounted on a cautery-tipped delivery system. Endoscopy 2015; 47: E619-620

[7] Tharian B, Varadarajulu S, Hawes R. Drainage of obstructed gallbladder with use of lumen-apposing metal stent. Gastrointest Endosc 2016; 83: $460-461$

[8] Anderloni A, Attili F, Carrara S et al. Intra-channel stent release technique for fluoroless endoscopic ultrasound-guided lumen-apposing metal stent placement: changing the paradigm. Endosc Intern Open 2017; 05: E25-29

[9] Veitch AM, Vanbiervliet G, Gershlick AH et al. Endoscopy in patients on antiplatelet or anticoagulant therapy, including direct oral anticoagulants: British Society of Gastroenterology (BSG) and European Society of Gastrointestinal Endoscopy (ESGE) guidelines. Endoscopy 2016; 48: 385-402

[10] Zuckerman M], Hirota WK, Adler DG et al. ASGE guideline: the management of low-molecular-weight heparin and nonaspirin antiplatelet agents for endoscopic procedures. Gastrointest Endosc 2005; 61: 189-194

[11] Săftoiu A, Ciobanu L, Seicean A et al. Arterial bleeding during EUSguided pseudocyst drainage stopped by placement of a covered selfexpandable metal stent. BMC Gastroenterology 2013; 13: 93

[12] Valats JC, Funakoshi N, Bauret P et al. Covered self-expandable biliary stents for the treatment of bleeding after ERCP. Gastrointest Endosc 2013; 78: $183-187$

[13] Aslinia F, Hawkins L, Darwin P et al. Temporary placement of a fully covered metal stent to tamponade bleeding from endoscopic papillary balloon dilation. Gastrointest Endosc 2012; 76: 911 - 913

[14] Loffroy R, Favelier S, Pottecher P et al. Transcatheter arterial embolization for acute nonvariceal upper gastrointestinal bleeding: Indications, techniques and outcomes. Diagn Interv Imaging 2015; 96: $731-744$ 